\title{
EREBEA
}

Revista de Humanidades

y Ciencias Sociales

Núm. 7 (2017), pp. 91-108

ISSN: 0214-0691

\section{«FAUCONS ET COLOMBES» SE LIBÉRER DE ROME OU CONVERTIR LE ROI?}

\author{
Marco Penzi \\ IHMC, Paris 1-Sorbonne
}

RESUMEN

Desde finales de 1589, los parlamentarios galicanos tomaron posiciones antiromanas: condenaron al legado Caetani por criminal de lesa majestad en 1591, acusaron al nuncio Landriano de hallarse ilegalmente en Francia bajo las órdenes del supuesto papa e incluso prohibieron toda comunicación con Roma. Los católicos regalistas, por otra parte, buscaron la pacificación o un acercamiento de ambas posiciones. Más adelante, será el mismo rey Enrique IV quien tome esta iniciativa. El presente artículo explicará el conflicto que enfrentó a los parlamentarios galicanos con la corte romana, entre los años 1589 y 1592, analizando las posiciones de los "halcones", opuestos a todo acercamiento, y esperanzados en crear en Francia una iglesia autocéfala, y las "palomas", más proclives a un mantenimiento de los vínculos sagrados con Roma.

Palabras Clave

Siglo XVI; Francia; Enrique IV; Galicanismo; Liga católica; Cisma.

Fecha de recepción: 12 de mayo de 2017 Fecha de aceptación: 18 de sept. de 2017
ABstract

Since the end of 1589 the Gallican members of Parliament took strong anti-roman positions: they condemned the legatus Caetani as guilty of lèse-majesté, then in 1591 they accused the nuncio Landriano to have illegally entered France upon the orders of a "supposedly" Pope; moreover they were forbidding any communication with Rome. The Catholic followers of the King on the other side were trying to achieve peace with Rome or at least a rapprochement. Later it will be Henri IV personally to take this initiative. This article will explain this conflict between the Gallican members of Parliament and Rome during the years 1589 and 1592, analysing the position of the "hawks" opposed to any rapprochement, and the hope to be able to build an autocephalous Church of France, and the initiatives of the "doves", more inclined to maintain the religious relationship with Rome.

\section{KEYWORDS}

Sixteenth century; France; Henri IV; Gallicanism; Catholic League; Schism. 

Depuis septembre 1585 et l'excommunication d'Henri de Bourbon, roi de Navarre et héritier présomptif au trône de France, par le pape Sixte $V$, une partie du Parlement parisien s'était ouvertement opposé aux interventions papales en France. L'excommunication ne fut jamais reconnue dans le royaume et le Parlement se refusa à la faire figurer dans ses registres : pour les parlementaires gallicans, le pape n'avait aucun droit d'excommunier un roi ou prince français, le cas de Jeanne d'Albret faisait école. Pour cette raison, si la Bulle romaine n'était pas signalée dans les registres du Parlement, elle n'avait jamais été reconnue en France, sa validité était nulle, sa portée anéantie. Cette opposition deviendra beaucoup plus importante et visible après l'assassinat d'Henri III et la succession de Navarre. Dès la fin de 1589, des positions anti-romaines étaient prises par les parlementaires gallicans : on condamnait le légat Caetani, envoyé en France par Sixte $\mathrm{V}$, comme criminel de lèse-majesté ${ }^{1}$. Au-delà des faits politiques et militaires relatifs à la guerre entre la Ligue et ses alliés et le parti soutenant Henri de Navarre, cette situation conflictuelle avec Rome, devait s'aggraver en 1591.

Pour caractériser les rapports entre la papauté et la Ligue en 1591, Pierre Matthieu, historiographe du roi, se limita à soutenir que le nouveau pape avait aidé militairement et financièrement les ligueurs ${ }^{2}$. Après la déclaration de Mantes de juillet, il remarquait qu'il "y eut un Arrest de la Cour de ce Parlement seante à Tours, sur les Bulles monitorialles de Grégoire, se disant pape : comme aussi [il] y en eut un autre de la Cour de Parlement de Chaalons au mois de Iuin de ladicte annee 1591 "'. Matthieu résume par ces quelques mots l'action pontificale favorable à la Ligue, mais aussi les arrêts des Parlements royalistes contre la politique de Grégoire XIV. Dans le contexte du récit de l'historiographe, la portée des arrêts de Chalons et Tours, semblait être un incident secondaire de la guerre civile.

Niccolo Sfrondato, milanais, élu pape le 5 décembre 1590, changea la politique romaine par rapport à celle de son prédécesseur Sixte V. D'une politique

$1 \mathrm{M}$. Penzi, "Tours contre Rome au début du règne de Henri IV”, in F. Gabriel, S. de Franceschi, A. Tallon, La culture gallicane. Références et modèles (droit, ecclésiologie, histoire), XVIe-XVIIe siècles, dans Revue de l'Histoire des Religions, $\mathrm{n}^{\circ} 3$ (2009), p. 329-347.

2 P. Matthieu, Histoire des derniers troubles de France, soubs les règnes des rois... Henry III... et Henry IIII avec un recueil des édicts et articles accordez par le Roy Henry III pour la réunion de ses subjects., s.l., 1606, livre V, f. $27 \mathrm{r}^{\circ}-\mathrm{v}^{\circ}$.

3 L'auteur ajoute que "le parlement de la ligue à Paris indigné de ce qui avoit esté fait à Chaalons, fit un Arrest contraire", dans Matthieu, Histoire..., p. 29. 
cherchant à unir les catholiques sous un candidat qui respectait la religion catholique et l'indépendance française face à l'Espagne, on passait à une politique interventionniste en faveur de la Sainte Ligue ${ }^{4}$. La nouvelle direction de la politique romaine devait se développer selon trois axes majeurs : l'exhortation des royalistes à rejoindre la Ligue ; l'envoi de troupes pour la guerre et d'un nonce extraordinaire pour pourvoir à la succession au trône de France ; enfin, l'excommunication pour ceux qui, en dépit des efforts romains, auraient continué à servir Navarre. Le nouveau cours de la politique papale faisait l'unanimité chez les ligueurs, au point que la Sorbonne remerciait le pape de l'aide "adversos sceleratos haereticorum et politicorum qui istis multo nequiores sunt ac Religioni pestilentiores" Pour parvenir à ses fins, la papauté envoyait tout d'abord en France une longue série d'écrits qui exhortaient les catholiques royalistes à abandonner Navarre. Par ailleurs, on organisait le financement et les préparatifs pour l'envoi d'une armée qui devait être assistée par un nouveau nonce, Marsilio Landriano, "référendaire des deux signatures ", délégué pour assister aux futurs États Généraux. Cette décision, qui avait été prise dès le 26 janvier 1591, entendait « remercier et encourager les uns, admonester et déterrer les autres"'. L'intention du pape était de dépêcher Landriano "pour réunir les nobles et les ecclésiastiques afin de leur faire connaître nos sentiments, en les excitant à pacifier le royaume et à défendre la foi catholique..." . Selon les instructions qu'il portait, le Nonce devait se montrer neutre vis-à-vis des deux factions : pour ce faire il aurait dû s'établir à Orléans, ville à

4 Le changement radical de la politique papale devint évident aussi pour les prélats romains, puisque, dans un Avviso du 29 décembre 1590, il était écrit : "Il dottor Briscia studia tuttavia de ordine del Papa, se egli puo con buona conscienza assolvere se stesso del giuramento fatto nella bolla delli millioni, che fece Sisto V per il disegno, che S.B. ha d'aiutare con essi la causa de cattolici ...”, dans Biblioteca Apostolica Vaticana (BAV), Mss. Urbinate 1058, p. 671; cf. L. von Pastor, Geschichte der Päpste im Zeitalter der katholischen Reformation und Restauration. Fribourg-Rome, Herder, 1958, vol. X, p. 544. Par ailleurs, on peut aussi comprendre le changement d'attitude de Rome à l'égard de la France au sujet des nominations des cardinaux Mandruzzo, Caetani, Santori et Facchinetti, tous pro-espagnols, dans la Congregatio in Rebus Franciae, la commission pontificale qui traitait les affaires de la France à Rome.

5 Archivio di Stato Vaticano (ASV), Segreteria di Stato Francia, t. XXXV, f. 55, lettre du $1^{\text {er }}$ mars 1591.

6 "Par ce mot, Monsieur d'Ossat veut dire, intimider, épouvanter, selon la signification du mot latin deterrere", dans Mr. Amelot de la Houssaie, Lettres du Cardinal d'Ossat. Paris : Jean Baudot, 1698, t. I : lettre IX (le 15 mars 1591), à la Reine Louise, p. 42.

7 Voir H. de L'Epinois, La Ligue et les Papes. Paris : Societé Génerale de Librairie Catholique, 1866., p. 471. L’envoi du nonce Landriano avait été largement annoncé en France, notamment par une lettre adressée aux Seize le premier mai $1591 ; c f$. ASV, Ep. ad Principes, 24, f. 348 ; ASV, Ep. Lat. Registra, Arm. XLIV, 35, f. 223v $\mathrm{v}^{\circ}$, ${ }^{\circ} 167$ : lettre de Grégoire XIV au Conseil des seize quartiers de la ville de Paris. Cf. P.-V. Palma-Cayet, Chronologie Novenaire. Choix de chronique et mémoires sur l'histoire de France avec notices biographiques par J. C. A. Buchon. Parigi, A. Desrez, 1836, p. 303304. Le duc de Montmorency en avait été informé le 19 avril : ASV, Ep. ad Princ., 24, f. 323r ${ }^{\circ}$; Également: ASV, Ep. Lat. Registra, Arm. XLIV, 35, f. $197 \mathrm{r}^{\circ}, \mathrm{n}^{\circ} 138$. 
mi-chemin entre Paris et la résidence des navarristes. Mais cette neutralité devait nécessairement s'appuyer sur le moyen de pression donné par l'armée pontificale et sur le soutien attendu du duc de Parme, commandant des troupes espagnoles envoyées pour se battre contre les hérétiques. En particulier, Landriano devait prêter serment au nom du Saint Père pour que le royaume de France ne soit jamais donné au roi d'Espagne.

Le nonce apportait une série de bulles et monitoires qui devaient être publiés en France : la bulle du $1^{\mathrm{er}}$ mars 1591 concernait les ecclésiastiques navarristes. Selon elle, tous les ecclésiastiques partisans de Navarre avaient l'obligation de l'abandonner, sous peine d'être suspendus et excommuniés sous quinze jours. Le monitoire était destiné à la noblesse, aux magistrats et au peuple français : il n'annonçait pas une immédiate excommunication, mais le vocabulaire utilisé laissait quand même imaginer que la sanction tomberait sur ceux qui auraient continué à suivre Henri de Navarre. Ce dernier était dénoncé en tant qu'excommunié, relaps, et privé de ses titres et seigneuries ${ }^{8}$.

La réaction des politiques ne tarda pas : le premier à intervenir fut le Parlement de Châlons le 10 juin 1591. L'arrêt émis à cette occasion commençait par une longue introduction historique. On y affirmait que la loi fondamentale pour la succession du royaume de France prévoyait le droit de primogéniture, qui avait été créé pour éviter "troubles \& divisions, qui autrement pourroient advenir : tellement qu'on peut dire qu'en France, le peuple n'être jamais un seul moment sans Roy, [...] comme le Roy en effect ne mourant point" ${ }^{\prime 9}$. Le texte précise qu'à

8 Les lettres du pape furent éditées à Rome, en latin, le $1^{\text {er }}$ mars 1591 : Literae S. mi D.N.D. Gregorii Divina Providentia Papae XIIII. Exhortatoriae \& monitoriae ad Principes, Duces, Marchiones, \& Comites, aliosq ; regni Franciae Proceres ac Nobiles, Henricii Borbonii quondam Navarrae Regis partes sequentes; Necnon ad Communitates, Universitates \& populos, atq ; caeteras personas seculares sub eiusdem Henrici dominatu existentes, ne eidem Henrico ulterius adhaereant, vel quoquomodo faveant. Romae, apud Paulum Bladum impressorem Cameralem, MDCXI. Ce texte fut traduit en français dès le 6 juin : Bulles de N.S. le pape Gregoire XIIII l'une contre toutes personnes Ecclesiastiques, suyvans le party de Henry de Bourbon, iadis Roy de Navarre. L'autre aux Princes, Seigneurs, Nobles \& autres personnes lä̈ques suyvans le mesme Party. Robert Nivelle et Rolin Thierry : Paris, 1591. Une autre version parut à Lyon le 24 juin : Lettres de nostre S. Pere le Pape Gregoire XIIII du nom, exhortatoires \& monitoires aux Princes, Ducs, Marquis, Comtes \& autres grands Seigneurs, \& Nobles du Royaume de France, suyvans le party d'Henry de Bourbon, iadis Roy de Navarre. Et aussi aux Communautez, Universitez, peuples, \& autres personnes seculieres qui sont sous la domination dudict Henry, à fin quils ne le suyvent d'avantage, \& ne le favorisent en quelque façon que ce soit. Lyon : Iean Pillehotte, libraire de la Saincte Union, 1591. Avec ces lettres monitoires, la Ligue rendait publiques les pouvoirs du nonce Landriano, lesquels devenaient ainsi une autre arme de propagande. Voir la Bulle de N.S.Père le pape Grégoire XIIII contenant les facultez données à Monseigneur le révérendissime Landriani, nonce apostolique au royaume de France. Paris : Robert Nivelle et Rolin Thierry, 1591; ou avec un autre titre: Les Facultez données par N.S-Père le pape Grégoire XIIII à monseigneur le reverendissime Landriano, nonce apostolique au royaume de France, Paris, 1591.

9 "Et en cela est la verité du mystère de la saincte Ampoulle, que l'antiquité a dit estre venuë du Ciel, non pas des sanctuaires des Papes, Archevesques, \& evesques, pour monstrer que la grace 
l'opposé du repos public qu'une telle loi devrait susciter, l'ambition de certains sujets, vassaux ou princes voisins, a pris depuis quelques années une telle ampleur que sous le "faux prétexte" de la religion, et grâce à l'aide du roi d'Espagne, ils ont conquis et enlevé au gouvernement du roi diverses villes du royaume. Mais Dieu a "mis le sceptre en la main de Henri IV, à present regnant, par la succession legitime de sainct Louys, $\&$ autres Roys ses predecesseurs $[\ldots]$ ". ${ }^{10}$. La révolte de ces sujets leur a permis également d'imprimer "certains petits libelles scandaleux en forme de bulles monitoires", qui sont directement contraires à la Loi fondamentale citée, à l'autorité des Rois de France, aux prérogatives et anciennes libertés de l'Eglise Gallicane, "abusans du nom du sainct siege Apostolique, comme si sa saincteté laissoit le nom de $S$. Pere \& pasteur, pour estre partial \& fauteur des rebelles et seditieux, contre le commandement du Sauveur du monde, de rendre à Cesar ce qui appartient à Cesar, \& à Dieu ce qui est à Dieu"11.

Ces affirmations du texte du Parlement royaliste, semblent condamner le pamphlet ligueur comme une supercherie. Dans le jeu opéré par les royalistes, Grégoire XIV, en tant que Saint-Père et pasteur des chrétiens, ne peut pas avoir publié un texte en faveur des rebelles qui s'opposent au roi légitime. Il s'agit nécessairement d'un faux, ce qui est confirmé par la suite du discours ${ }^{12}$. Si ces phrases laissent croire à une position "conciliante", voire indulgente, envers Rome, la suite du texte se révèle être une attaque directe contre l'autorité pontificale. Toujours en jouant sur un registre hypothétique, qui est en réalité un acte accusatoire, le texte du Parlement affirme que la faction espagnole a désormais

de Dieu, dont nos Roys sont honorez, \& par laquelle ils regnent, vient immediatement de la seule providence, sans dépendre des hommes, quels qu'ils soient", dans Arrest de la cour de parlement seant à Chaalons, sur certains libelles iniurieux et scandaleux, intitulez, bulles monotoriales, et imprimés a Rheims, 1591. Ce texte est aussi présent dans P. Dupuy, Preuvez des libertez de l'Eglise Gallicane. Paris : Sebastien Cramoisy \& Gabriel Cramoisy, 1651, t. I, chap. IV, p. 37. Cette phrase, brillant résumé de la doctrine de la monarchie de droit divin, est en réalité une réponse directe aux introductions historiques que Grégoire XIV avait placées en tête de ses monitoires adressés aux ecclésiastiques et nobles français. Cf. Bulles de N.S. Pere le pape Gregoire XIIII...

10 Dupuy, Preuves, t. I, ch. IV, p. 37.

11 "et contre le precepte de $S$. Pierre mesme, qui a commandé, $\&$ presché de son temps, d'honorer les Roys, ores qu'ils fussent lors notoirement payens [...]", dans Dupuy, Preuves..., p. 37.

12 "ou comme s'il vouloit venir (le Pape), à main armée, au partage de la dissipation qu'ils pretendent faire de cette couronne, au lieu de prendre la voye de la douceur, mieux seant à son estat, pour instruire \& edifier un Roy, qui s'est toujours autant disposé à recevoir conseil \& instruction, comme ses ennemis à lui en oster le loisir, de peur de voir la paix \& la justice restablie en France, en laquelle il faudrait rendre compte de leurs vies \& actions ...", dans P. Dupuy, Preuves..., p.37. Le Parlement de Châlons, jouant sur l'impossibilité de l'appui papal aux séditieux ligueurs, change les données historiques. Dans cette fiction, qui veut que le pape ne puisse pas être un suppôt de la politique de la Ligue, Henri IV devient un roi qui ne demande qu'à être instruit. La fiction parlementaire atteint ici son paroxysme : les juristes de Châlons, étaient bien au courant des doutes d'une partie de la noblesse catholique. 
tout pouvoir dans Rome ; de son côté, “a France n’est destituée des remedes ordinaires d'appel comme d'abus, \& autres, par le moyen desquels telles entreprises seront reprimees..."13. En vertu de ses remèdes ordinaires, la cour de Parlement déclare "lesdits libelles, iniurieux, scandaleux, \& diffamatoires, tendans à sedition, \& à l'eversion de l'estat, couronne, \& maison de France, pour y introduire l'estranger..." 14 . Les textes des bulles devront être lacérés et brûlés, et tout sujet qui favorisera de quelque manière de telles publications sera jugé en tant qu'ennemi et perturbateur du repos public.

Henri IV et le Parlement de Tours répondirent aussi aux bulles pontificales : le premier, par une déclaration du 4 juillet 1591 ; le deuxième, par un arrêt contre "la partialité du pape et de son légat". Les raisons qui poussèrent Henri et les Politiques à répondre au pape : "l'un avec la modération d'un politique, l'autre avec une violence qui ne laissait rien à envier aux pires énergumènes de la faction adverse", doivent donc être cherchées dans l'exigence de réagir à ce qui était conçu comme une attaque de la papauté ${ }^{15}$. Dans sa déclaration de Mantes du 4 juillet, Henri IV s'adresse directement au peuple français. Le roi soutient avoir toujours travaillé à la paix, espérant une solution du conflit religieux à travers un concile “par lequel ce qu'il y a de different $\&$ discordant au faict de la religion, peust estre si bien esclaircy $\&$ vuidé, qu'il ne peust jamais plus estre en aucune dispute $\&$ incertitude" ${ }^{16}$. Il insiste sur le fait que les divisions du royaume ne sont que le fruit des ambitions de tous ceux qui, avec l'appui de l'Espagne, de la Savoie et de la Lorraine, veulent diviser l'État, pour y installer des gouvernements personnels. La religion n'est que le prétexte de leur soulèvement, à travers lequel ils ont abusé de la crédulité des derniers papes, "mais cette leur malice fut bien-tost découverte par le feu pape Sixte, que l'on a veu en ses derniers jours se repentant d'avoir esté par eux abusé, bien resolu de fulminer contre eux rigoureusement, \& plus qu'à leur instigation ils n'avoient auparavant fait contre d'autres" ${ }^{17}$.

Jusqu'ici Henri de Navarre ne fait que reprendre la polémique et la propagande des Politiques. Cette reconstruction des événements culmine avec une description de la politique de Grégoire XIV. Celui-ci, en étant trop crédule, s'était fait convaincre par les ligueurs, sans consulter qui que ce soit. De cet abus commis par les ennemis du royaume découlait l'envoi du nonce, entré clandestinement dans le royaume, et qui portait plusieurs monitoires contre les ecclésiastiques et les nobles qui suivaient le roi de Navarre. Henri enjoint alors à ses Parlements

13 Dupuy, Prenves..., p.37.

14 Dupuy, Prenves..., p. 38.

15 E. Maugis, Histoire du Parlement de Paris, Genève, Slatkine-Mégariotis, 1977, t.2, p. 78.

16 Lettres patentes du Roy, contenans declaration de l'intention quil a pour maintenir l'Eglise \& Religion Catholique, apostolique \& Romaine en ce Royaume, ensemble les droicts \& anciennes libertez de l'Eglise Gallicane, dans Dupuy, Preuves..., p. 38.

17 Dupuy, Preuves..., p. 38. 
de procéder, pour la défense des "Libertez de l'Eglise Gallicane", contre le nonce sans perdre plus de temps ${ }^{18}$. Il exhorte aussi les cardinaux, archevêques et évêques à se réunir pour prendre des mesures appropriées selon le droit et les "libertez gallicanes" contre les sanctions prises par le pape et le nonce. Le 5 août, suivant l'ordre du roi, le Parlement de Tours déclarait dans un arrêt les bulles pontificales "nulles, abusives, seditieuses, damnables, pleines d'impieté $\&$ d'impostures, contraires aux saincts Decrets, droits, franchises, \& libertez de l'Eglise Gallicane ..."; elles devaient être lacérées et brûlées, et il était défendu aux ecclésiastiques, sous peine d'être accusés de crime de lèse-majesté, "d'en publier aucune copie" et à tous d'y obéir ${ }^{19}$. La Cour dans un crescendo belliqueux "a declaré, $\&$ declare Gregoire, soy disant pape XIV de ce nom, ennemy de la paix, \& de l'union de l'eglise Catholique, Apostolique, \& Romaine, du Roy, $\&$ de son Estat, adherant à la conjuration d'Espagne, $\&$ fauteur des rebelles, coupable du très cruel, très-inhumain, \& très-detestable parricide, proditoirement commis en la personne de Henry III de très-heureuse memoire..." ${ }^{20}$.

Le Parlement imputait à Grégoire plusieurs crimes : l'usurpation du titre de pape ("soy disant pape") ; l'opposition au Roy et au royaume et fauteur des rebelles; l'hostilité à "l'union de l'Eglise Catholique, Apostolique, \& Romaine", c'est-à-dire schismatique ; et le crime de l'assassinat d'Henri III. Le texte des parlementaires est très adroit : c'est le pape qui s'est détachée de la catholicité ; qui a abandonné les véritables catholiques; qui s'est séparée de l'ensemble des fidèles. On accusait le pape d'être schismatique, de diviser les catholiques pour des raisons politiques et non religieuses. Le Parlement opère une inversion des rôles. Celui-ci, en défenseur zélé des "Libertez Gallicanes", devenait le porte-parole de "l'Eglise Catholique, Apostolique, \& Romaine" contre la partialité du pape romain. L'arrêt prévoyait aussi des décisions spécifiques : "a inhibé \& defendu, sur semblable peine (lèse-majesté), à tous Banquiers, respondre, ou faire tenir lettres de Banque à Rome, or ny argent, pour avoir Bulles, provisions, $\&$ dispenses, $\&$ autres expeditions quelconques : \& si aucunes sont obtenües, aux Juges d'y avoir egard"21. L'arrêt de Tours déclarant Grégoire XIV schismatique défend aux ecclésiastiques et aux laïcs français tout commerce vénal ou spirituel avec Rome. La suite de l'arrêt concernait les dispositions à prendre contre "Marsilius Landrianus, soy disant Nonce dudit Gregoire" qui devait être constitué prisonnier en vue de

18 Dupuy, Preuves..., p. 39.

19 Arrest du Parlement seant à Tours, donné sur l'appel comme d'abus interjecté par le procureur General du Roy, de l'octroy \& execution de certaines Bulles émanées de Rome. Du Lundy cinquiesme Aoust 1591, dans Dupuy, Preuves..., p. 41.

20 ibidem.

21 ibidem. 
son jugement ${ }^{22}$. Le Parlement de Tours, en suivant la lettre d'Henri IV, ordonnait aux ecclésiastiques français de réunir un concile qui pût trouver un dénouement.

Selon Édouard Maugis, le Parlement de Châlons, dans son premier arrêt, avait utilisé un langage juridique. Le Parlement de Tours, qui avait nettement durci le ton, revenait "aux outrances d'un Pierre Flotte et de Philippe le Bel"23. Duplessis-Mornay semble donner "sa" solution aux questions précédentes, quand il s'exprime au sujet du Parlement de Tours : "ceste court ... a donné un arrest contre Gregorie pretendu pape, a annullé son excommunication, l'a declaré schismatique, ordonné que sa bulle fust bruslée, desfendu sur peine de crime de leze majesté de s'adresser plus à lui. Nul ne doubte qu'elle ne l'a faict competemment, et selon sa jurisdiction ; car soubs Philippe Auguste, Philippe le Bel, Charles V, VI et VII, Louis XII, en pareilles matieres, ont esté donné pareil arrests, et par ces mesmes juges. Elle ne faict donc rien de nouveau, et n'entreprend rien qui ne soit sien $[\ldots]^{\prime 24}$.

L'arrêt de Tours, suivi par d'autres arrêts de Parlements royalistes, ne pouvait pas passer inaperçu auprès des polémistes ligueurs. Le 24 septembre 1591, le Parlement ligueur de Paris répondit à l'acte donné à Tours ${ }^{25}$. La Cour déclarait n'avoir autre "intention et ne tendant à autre fin que de maintenir la religion ancienne, Catholique, Apostolique et Romaine, et l'ordre estably en l'esglise de Dieu, et sopporter et resister de tout son pouvoir à l'introduction de l'heresie, en ce tres chrestien Royaume et conserver l'Estat Royal en son entier". Pour cette raison, elle annulait et défendait à tous d'obéir à l'arrêt donné à Tours ${ }^{26}$. Le texte parisien ordonnait à tous les Français de :

porter honneur respect, et reverence et obeissance à nostre sainct pere le pape Gregoire XIIII à present tenant le sainct siege apostolique, comme vicaire de Dieu en terre, successeur de sainct pierre et chef universel de ladicte esglise, honorer, reverer, crainde et redouter ses dites bulles monitoires procedantes de sa pieté, dilection, providence et sollicitude paternelle, ensemble recognoistre ledict nonce en sa charge et facultez à luy octroiées suivant la verification d'icelles

22 Suivent les dispositions pour la publication de l'arrêt dans la ville de Tours, bailliages et sénéchaussées et pour notification aux évêques et archevêques et à l'ensemble des ecclésiastiques.

23 Maugis, Histoire..., t. 2, p. 80.

24 P. Duplessis-Mornay, Mémoires et correspondance, Genève, Slatkine-Reprints, 1969, t. V, p. 90-91: Despeche envoyee de Tours au Roy, du 3 octobre 1591.

25 Arrest de la cour de Parlement contre certain pretendu Arrest donné à Tours, sur le faict des bulles monitoires de nostre sainct père. Paris : chez Robert Nivelle, 1591. Voir également aux AN (Archives Nationales) X1A 1711, f. 219, le 23 septembre, et F.-A. Isambert et alli (éd.), Recueil général des anciennes lois françaises. Paris : Belin-Leprieur, 1829, vol. XV, p. 32.

26 Arrest de la cour de Parlement, p. 4. 
faictes en ladite court, le tout sur peine contre les contrevenants d'estre punis comme ennemis de Dieu, perturbateurs de la paix et union de son esglise ${ }^{27}$.

Les magistrats parisiens déclaraient les signataires du texte de Tours coupables de lèse-majesté divine et humaine. Édouard Maugis, pour qui l'arrêt de Tours n'est autre chose qu'un factum, soutient qu'il n'est guère surprenant que "la Cour de Paris ait condamné ce factum avec plus de solennité que celui de Chalons, exigeant, cette fois, la signature de tous ses membres, présents ou empêchés, au bas de son arrêt de révocation du 18 septembre". Pour cet auteur, l'arrêt parisien ne fait que traduire la "stricte doctrine catholique, sans la réserve suffisante des droits de la couronne"28. L'échange "d'arrêts furieux", selon une expression du chancelier Cheverny, ne suffisait pas à résumer les positions des partis en lutte ${ }^{29}$. Des Cours de Parlement, l'affrontement allait passer aux pages des nombreux libelles édités par les deux partis ${ }^{30}$.

Chez les royalistes, l'historien Palma Cayet soutient qu'on pouvait compter entre les "commonitoires et excommunications du pape jusqu'à vingt-six nullités" ${ }^{31}$. D'autres auteurs tels Guy Coquille, François de Claris et l'anonyme du Francophile condamnaient les prétentions papales à la suprématie du spirituel sur le pouvoir temporel, accusant le pontife d'avoir falsifié ou utilisé sans discernement les Saintes Écritures. Ces raisons étaient suffisantes pour démontrer la partialité du pape et donc pour invoquer le concile général. Dans la déclaration de Mantes, Henri IV invitait les prélats de son parti à se réunir en assemblée "afin de mettre eux $\&$ le Clergé à couvert des Bulles de Rome" 32 . Le ton des arrêts du mois d'août 1591 avait suscité "des peurs" au sein des ecclésiastiques du camp du roi : ils croyaient que les cours souveraines préparaient un procès contre Grégoire XIV. Ces peurs des ecclésiastiques furent transmises aux parlementaires. Ces derniers répondaient que l'arrêt (de Tours) n'était pas destiné à la personne du pape, mais

27 ibidem.

28 Maugis, Histoire..., t. 2, p. 80.

29 Philippe Hurault, Mémoires. Paris : Desrez, 1838, p. 277.

30 A. Maillard, Le francophile pour tres-grand, tres-chrestien, tres-magnanime \& tres-belliqueux Prince Henry Auguste IIII. Roy de France \& de Navarre. Contre les conspirations du Roy d'Espagne, du Pape \& des rebelles de France, s. l., 1591 ; L. Servin, Vindiciae secundum libertatem ecclesiae gallicanae, et regii status gallofrancorum. Sub Henrico III rege Francorum et Navarrae. Augustae Turonum : ex officina J. Metateri, 1591; G. Coquille, Discours des droits ecclésiastiques et libertés de l'Eglise gallicane, 1591 ; Matteo Zampini, Ad calumnias et imposturas a pseudo parlamentis Cathalaunensi et Turonensi ac Carnotensi Conventiculo, ad Catholicae Religionis perniciem populique deceptionem, impie confictas in Gregorium XIIII illiusque monitionis literas, ad Clerum, Prijncipes, Nobiles, et Populos Franciae. Responsio Matthaei Zampini Recanatensis. Paris : Rolin Thierry, 1591.

31 Palma-Cayet, Chronologie Novenaire...., p. 318.

32 J.-A., De Thou, Histoire universelle. Londres : s.n., 1734, t. XI, 1. CI, p. 372. 
avait été pris uniquement contre ses bulles. Pour certains membres du Parlement, les temps étaient désormais mûrs pour montrer que la France ne tolérait pas les abus romains sans régir de conséquence : "le vray moyen de réduire le pape à la raison, c'est de lui montrer les dents. Nous avons assez éprouvé que les remèdes doux ne font que rengréner notre maladie" 33 .

L'assemblée du clergé, ordonnée par Henri IV et ensuite par les Parlement, s'ouvrait à Mantes en septembre 1591. Transférée à Chartres, elle se terminait avec la signature d'un document officiel par les ecclésiastiques présents ${ }^{34}$. Le texte écrit par les prélats tenait à la fois du reproche formel à un pape injuste et de la lettre de fidèles sujets qui voulaient qualifier et expliquer leurs actes à leur seigneur ${ }^{35}$. Les prélats déclaraient que le pape "mal informé de l'estat des affaires de ce Royaume, \& de nos deportemens", aurait envoyé en France des monitoires et excommunications contre les ecclésiastiques et la noblesse, à cause des artifices des ennemis de l'État. Le pape, trompé par les ligueurs, aurait donc agi contre les libertés gallicanes qui prévoyaient que le Roi et ses Parlements fussent intouchables par l'excommunication. Le souverain Pontife, mal informé, aurait sans en avoir les compétences et le pouvoir, produit des actes contraires à la Loi (Liberté) de l'Église gallicane. Pour ces raisons, les prélats français, déclaraient les bulles "fort nulles tant en la forme, qu'en la matière, injustes \& suggerées per les artifices des estrangers, ennemis de la France, \& qu'elles ne nous peuvent lier, ni obliger, ni autres françois catholiques estans en l'obeissance du roy" ${ }^{36}$. De même, "sans entendre rien diminuer de l'honneur, \& respect deu à nostre sainct Pere", les ecclésiastiques afin d'éviter que les fidèles ne soient "circonvenus, abusez, ou divertis de leur devoir envers leur Roy, \& leurs Prelats”, se réservaient le droit d'envoyer

33 Lettre de Charles Faye à monseigneur de Bellièvre, 22 août 1591, dans E. Halphen, Lettres inédites de Jacques Faye et de Charles Faye. Paris : librairie des bibliophiles,1880, p. 116-118.

34 Les Cardinaux, Archevesques \& Evesques, Abbez, Chapitres, \& autres Ecclesiastiques, convoquez \& assemblez à Mantes, \& depuis à Chartres, pour adviser \& pourvoir aux affaires de l'Eglise. (21 septembre 1591) A tous les estats, Ordres, Villes \& peuples catholiques de ce royaume, Salut., dans Dupuy, Preuves, v. II, p. 45. Les signataires étaient : Charles, cardinal de Bourbon, (il s'agit de Vendôme, qui avait repris le titre de son oncle décédé en 1590, sans qu'aucune disposition ne lui ait été notifiée par Rome), Philippe cardinal de Lenoncourt, Renaud de Beaune archevêque de Bourges, Philippe du Bec évêque de Nantes, Nicolas de Thou évêque de Chartres, Nicolas Fumée évêque et comte de Beauvais, pair de France, Henry d'Escoubleau, évêque de Millezais, Claude D’Angennes, évêque du Mans, Cosme Classe, évêque et comte de Châlons, pair de France, René de Daillon nommé à l'évêché de Bayeux, Jean Touchard abbé de Belosane, Jacques David du Perron, Claude Govin doyen de Beauvais.

35 Le texte de la déclaration commence ainsi : "L'apostre parlant aux Pasteurs de l'eglise : Prenez, dit-il, garde à vous, \& à tout le troupeau sur lequel Dieu vous a establis, pour regir $\&$ gouverner son Eglise, qu'il a acquise pas son sang. Ce que reconnaissans estre de nostre devoir, \& de ne souffrir les ames Chrestiennes, qui sont soubs nostre charge, se divertir les loix, \& commadement de Dieu", dans Dupuy, Prenves..., v. II, p. 45.

36 Dupuy, Preuves..., v. II, p. 45. 
une députation vers le Saint-Père pour lui faire entendre leurs raisons. Les prélats concluaient leur écrit en invitant les catholiques à s'unir dans les prières pour la conversion du roi et l'union de l'Église catholique, apostolique et romaine ${ }^{37}$.

Au sein du parti du roi, deux positions politiques et religieuses différentes s'affrontent. Nous avons évoqué la véhémente action des parlementaires navarristes contre les bulles de Grégoire XIV. L'opposition totale, appuyée par les protestants à Rome, ouvrait un schisme dans l'Église catholique. Les "faucons" gallicans se lançaient contre le pape et sa politique en utilisant une arme ancienne, mais extrêmement efficace. La rupture avec Rome était complète, et elle suscitait l'approbation des huguenots, et en particulier de Duplessis Mornay. Dans un contexte tout différent, le schisme avec Rome avait montré sa validité mais surtout sa faisabilité : comme en Angleterre sous Henry VIII, la création d'une Église séparée de Rome, avec à sa tête le roi, semblait possible. Navarre d'abord et son Parlement, par la suite, avaient sommé le clergé de convoquer un concile devant déboucher sur une séparation définitive avec Rome.

L'autre courant politique était formé par le clergé catholique. Celui-ci suivait Navarre et avait reçu l'ordre de se réunir, mais ses délibérations n'allèrent pas dans la direction voulue par le roi et le Parlement. Les "colombes", réunies à Chartres, en réaffirmant leur soutien à la politique du roi et de son Parlement, essayèrent de trouver un point d'accord avec Rome, ou bien de solliciter une procrastination en vue d'une séparation définitive de l'Église catholique. L'envoi d'une députation au pape, pour lui exposer la situation française, constituait un refus du schisme et de la rupture avec le catholicisme, un rameau d'olivier présenté au roi scellant la paix entre la couronne et Rome.

Le concile de Chartres était un échec pour les "faucons" partisans d'une rupture avec Rome. Dans le même temps, on ne peut pas le considérer comme un succès pour les "colombes" : ils avaient les mains liées et avaient besoin de l'approbation royale pour envoyer des émissaires à Rome. Corollaire du texte du clergé réuni à Chartres : la décision fut prise d'envoyer une députation au roi de Navarre $^{38}$. Celle-ci fut reçue le 5 novembre, et les jours suivants, par Henri IV à Noyon. De cette députation nous possédons un précieux procès-verbal ${ }^{39}$. Les ecclésiastiques choisirent Renaud de Beaune comme porte-parole.

37 ibidem.

38 Les prélats royalistes fonctionnaient telle l'assemblée du clergé de France, en envoyant une députation au roi. De cette délégation nous possédons un ample Procès verbal des Deputez de l'Assemblée du Clergé de Chartres, envoyez vers le Roy le 5 novembre 1591. Dans Dupuy, Preuves..., v. II, p. 45-50. La députation était composée par l'évêque de Beauvais, Renaud de Beaune, les évêques du Mans et d'Angers, les Doyens de Tours et Beauvais, François Choisnyn Chanoine du Mans, Pierre Cibot docteur en théologie, chanoine et syndic de Limoges, tous députés de l'assemblée réunie à Chartres.

39 Dupuy, Preuves..., v. II, p. 45-50. 
Si, d'un côté, les députés du clergé devaient se justifier auprès du roi de n'avoir pas voulu affronter les questions qui leur avaient été posées par les Parlements et le monarque, de l'autre, les prélats avaient particulièrement à cœur d'informer le souverain de la nécessité d'une ambassade à Rome. En effet, sans connaissance des volontés du pontife, l'assemblée aurait pu statuer avec précipitation et se départir du Saint-Siège, chose terrible qui aurait conduit le royaume au schisme. Selon les prélats, cela aurait amené également les sujets à se départir de leur souverain, en prenant l'exemple du clergé comme excuse ${ }^{40}$. Henri IV, dans sa réponse du 17 décembre 1591 au sujet du voyage à Rome qui était la requête principale du clergé, dont il était clair qu'elle allait à l'opposé des décisions de l'arrêt de Tours, écrivait : "[...] qui est le plus douteux, je desire de m'en resoudre avec autres ; j'attends messieurs de Montpensier \& de Nevers, $\&$ les Presidens de Tours et de Caën, qui ont des remonstrances à me faire, $\&$ prie de ne resoudre ce qu'ils ont à me dire, ou faire entendre sans les avoir ouys" ${ }^{\prime 1}$.

Le Parlement de Tours s'opposa fermement à une telle ambassade, rappelant le sens de l'arrêt donné en août et qui prohibait la communication avec Rome, Grégoire XIV ayant été déclaré "ennemi du Royaume" : la décision fut donc ajournée ${ }^{42}$. Dans sa réponse, le roi se montre également concerné par les préparatifs de guerre des armées de la Ligue et des troupes papales, et se réfère à ce sujet à Grégoire XIV : “je l'estimerai comme un prince étranger qui me fait la guerre, auquel je ne veux mes sujets avoir recours, ni aucune communication avec lui. C'est chose de laquelle j'ai advisé avec Monsr le Chancelier et avec les autres et non avec vous, mon cousin (Henri parle ici du/au cardinal de Bourbon); car je vous ai estimé être du nombre de ceux qui m'ont fait ces requêtes et remontrances, pour ne vous point admettre à ce Conseil. Vous avez été de l'assemblée et y avez présidé, et est le tout passé avec vous...”³. Suite à l'entrevue, le cardinal de Bourbon préconise que pour arriver à une solution souhaitable concernant les relations avec la papauté, il serait plus convenant de ne pas laisser "MM. de Parlement entreprendre sur l'état de l'Église, ni d'en connaître, hors des procès comme d'abus - en la moisson de laquelle Sa Majesté ne permettra, s'il lui plait, qu'ils mettent leur faux, non plus que les ecclésiastiques mettent la leur en la

40 "Autrement, si cet affaire eust precipité, n’y a doute que de la precipitatio on n'eust jugé que l'assemblée fust departie du S. Siege, \& de son exemple le subjet se pouvoir substraire de l'obeyssance de son prince, l'inferieur se licencier se son superieur pour fautes par eux faites, \& par ce moyen planter un schisme autant pernicieux, qu'est l'unité salutaire, au lieu de laquelle consiste le salut, \& lequel conservé en son entier, rend les forces $\&$ astuces de l'ennemy vaines $\&$ sans nuisance", dans Dupuy, Preuves..., p. 46.

41 Dupuy, Preuves..., p. 49.

42 De Thou, Histoire..., t. XI, liv. CI, p. 375-376.

43 Dupuy, Preuves..., p. 49; Maugis, Histoire..., t.2. p. 82-83. 
moisson desdits Sieurs de Parlement" 44 . Le cardinal et Henri IV discutent en effet d'un point bien précis : le prélat est à l'origine du texte émanant de l'assemblée de l'Église royaliste et termine son discours en rappelant au roi le problème au cœur de l'assemblée. Il s'agissait de recoudre la déchirure avec Rome, provoquée par les arrêts du Parlement.

Le concile invoqué par Henri IV et le Parlement de Tours semble avoir intéressé beaucoup plus les contemporains que les historiens des guerres de religion. Ce concile a été souvent traité comme un événement mineur de l'histoire de la période : idée tirée probablement de l'importance que les historiens de l'époque ont conférée à l'évènement. Palma Cayet en est l'exception. Selon cet historien : "ceste assemblée fut celebre, et s'y trouva nombre de prelats et ecclesiastiques de divers endroicts de la France, et mesmes plusieurs archevesques et evesques des villes de l'union, car peu se trouverent des villes de ce party qui ne chasserent

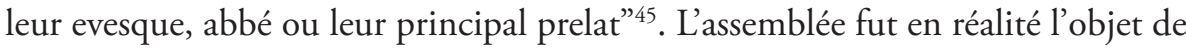
vibrantes discussions.

La Ligue réagit aux arrêts des Parlements royaux mais aussi au texte produit par les prélats rassemblés à Chartres. Le premier octobre un texte de Matteo Zampini fut imprimé, avec le titre Ad calumnias et imposturas a pseudo parlamentis cathalaunensi et turonensi ac carnotensi conventiculo... ${ }^{46}$. Le texte est divisé en quatre parties : les trois premières sont dédiées à la réfutation des arrêts de Châlons, Tours et de la déclaration de l'assemblée de Chartres. La quatrième partie est par contre une étude critique des théories gallicanes, longue de 250 pages $^{47}$.

Chez les royalistes, des objections et des doutes se levèrent aussi sur l'opportunité de ce concile ou conférence et sur sa légitimité même. L'exemple le plus clair est donné par Duplessis-Mornay qui exprime sa perplexité dans une lettre adressée à Henri IV ${ }^{48}$. Le pape des huguenots soutenait que rien de bon ne pouvait sortir de cette réunion de prélats catholiques. Il contestait aussi la

44 Dupuy, Preuves..., p. 49.

45 Palma Cayet, Chronologie novenaire..., p. 319.

46 M. Zampini, Ad calumnias... ; La première partie de ce texte du juriste italien fut traduite en français avec le titre Défence pour les bulles monitoires de N.S.-P. le pape envoyées aux ecclésuiastiques, princes et nobles, suivans le navarraois, contre les perverses intentions et petiferes vomissemensdes imposteurs politiques, qui falsement se disent iuges et parlment de Chaalon (Paris : Robert Nivelle, Rolin Thierry, 1591). Raybaud qui a consacré quelques pages à l'analyse du pamphlet le trouve : "[...] verbeux et incohérent. L'absence d'un plan véritable rend peu perceptible la progression de la démonstration ", mais il lui reconnaît une certaine dignité du point de vue des idées", dans L.-P. Raybaud, "La royauté d'après les oeuvres de Matteo Zampini", C. Bontemps, L.-P. Raybaud, J.-P. Brancourt (éd.), Le prince dans le France des XVIe et XVIIe siècles. Paris : PUF, 1965, p. 187-201.

$47 \mathrm{La}$ quatrième partie porte un titre spécifique : Confutatio Praetensionim quibus potissimum, Pseudum-Parlamentum Turonense, \& Carnolense Conventiculum, schisma construens, Regnum Franciae, \& Ecclesiam gallicanam, a summi Ponteficis, obedientia divertere : atque ab ecclesia catholica separare, nituntutr.

48 Duplessis-Mornay, Mémoires et correspondances, p. 85-97. 
légitimité de certaines décisions de l'assemblée, comme celle qui prévoyait l'envoi d'une députation, ou d'un envoyé à Rome, décision qui était contraire, comme nous l'avons vu, à la défense prononcée par l'arrêt de Tours. Mornay remarquait aussi qu'un des articles de ladite assemblée prévoyait que l'on ne soumettrait plus à un jugement du Parlement "des differends entre le pape et le roy, la puissance par lui prestendue et les privileges du royaulme" 49 . C'était comme demander l'abolition de toute future décision des cours de justice sur des questions dites de "religion » ou à caractère ecclésiastique. Un autre pamphlet émanant de la Ligue était publié entre la fin du mois de septembre et début octobre 1591 ; il critiquait aussi les résolutions prises par l'assemblée ecclésiastique de Chartres ${ }^{50}$. Le pamphlet porte une attaque personnelle aux prélats présents aux discussions. L'auteur expose des faits qui stigmatisent chaque représentant du clergé royaliste. Le but est clair : diminuer la valeur des personnalités présentes pour réduire la légitimité de la réunion. Le cardinal de Bourbon, par exemple, est montré du doigt car il porte le titre de cardinal alors qu'il n'a pas encore pris les ordres ecclésiastiques. Au cardinal, président de l'assemblée, on reproche sa conduite envers la religion catholique :

Vous faites bien tost passer sans basteau la pauvre France en Angleterre, et nous transformez en heretiques Lutheriens Allemans, n'ayans que femmes ou laics pour chefs de leur pretendue Eglise et religion. Car du cardinalat, il n'en a que le bonnet, lequel il change souvent à un hausse col ou un casque, n'estant encore resolu s'ils era sacré ou profane. Il feroit donc mieux d'etudier aux peines deues tant de ce siecle qu'en l'autre, à ceux qui laissent ou font mourir leurs parents en captivité pour les eviter, que conspirer ainsi avec vous par un autre parricide contre l'Eglise sa mere et nourrice ${ }^{51}$.

L'auteur de ce texte ligueur conteste l'autorité et la légitimité de l'assemblée. Le concile avait en effet été convoqué par le roi de Navarre, un hérétique, relaps et excommunié qui ne possédait pas l'autorité nécessaire à la convocation d'une assemblée d'ecclésiastiques catholiques. L'auteur observe aussi que le nombre limité de prélats présents ne pouvait être considéré comme adapté à la prise des décisions qui auraient dû avoir un caractère national mais aussi universel. Le cardinal de Bourbon ne pouvait d'ailleurs pas prendre la présidence d'une telle

49 Duplessis-Mornay, Mémoires et correspondances, p. 86.

50 Responses aux articles du conciliabule tenu à Chartres par aucuns prélats politiques, le 21 septembre 1591. Dédié à eux-mesmes, par H.D.V.I.C. Paris : Guillaume Bichon, 1591. L’auteur définit son écrit comme un "eschatillon de responce", attendant une "belle et docte pièce qu'on vous prépare en latin”: il s'agit probablement du texte de Zampini édité le premier octobre.

51 idem, p. 4. 
assemblée, comme en réalité il l'avait fait car il n'était pas diacre, ni prêtre. Ce faisant il contrevenait aux canons des apôtres, aux décisions du concile de $\mathrm{Ni}$ cée et à bien d'autres concernant les réunions et assemblées ecclésiastiques. Le texte examine par la suite les décisions prises par l'assemblée de Chartres point par point. Selon l'auteur du pamphlet, les ecclésiastiques qui étaient présents auraient commis des fautes graves : 1) ils ont reconnu comme souverain un hérétique, qui n'a pas le droit d'être roi au vu de son excommunication, de sa non-appartenance à l'Église, des lois fondamentales etc.; 2) les ecclésiastiques présents auraient parjuré : leurs décisions s'opposent en particulier au serment prêté par l'assemblée des États en 1588 au sujet de la catholicité du Royaume ; 3) ces mêmes prélats auraient invité des catholiques à prier pour un hérétique, appelé roi ; 4) ce dernier point est d'ailleurs emblématique d'une situation de fait que les présents essayent d'imposer à l'ensemble des croyants : ils exhortent à prier pour un excommunié qui n'aurait réservé aux catholiques autre chose qu'un sort ou traitement similaire à celui que les catholiques subissaient en Angleterre; 5) le texte rédigé par les prélats, pour les raisons énumérées, ne serait autre chose qu'une supercherie vouée à duper les simples ; 6) non sans malice, l'écrivain ligueur constate que les prélats présents assurent que le roi de Navarre conservera la foi des catholiques et leurs possessions, mais on a vu se produire aussi tout le contraire dans des places telles que La Rochelle, Nérac etc., où les huguenots, après avoir fait des promesses similaires, ont chassé les catholiques et la religion. En dernier ressort, l'auteur utilise l'actualité pour montrer les réelles intentions des Politiques et leur conduite envers les catholiques romains : "Ce sera comme il fait maintenant à l'evesque d'Evreux (lequel après avoir esté traicté avec toute barbarie ils ont fait mourir de poison) et à tous ce qui n'adhérent à sa tyrannique impiété [...]"52.

\section{Conclusion}

Nous avons longuement traité d'une polémique juridictionnelle. Elle pourrait sembler marginale à l'histoire de la Ligue, elle a d'ailleurs été en partie considérée comme telle. Les historiens ont souvent rappelé les années 1591-1592 comme des années de guerre, d'importants faits militaires, de polémiques stériles, de simples guerres de pamphlets sur la base de la reconquête du royaume de la part d'Henri IV. Comme nous venons de le montrer, la situation politique mais aussi religieuse du royaume de France ne peut pas être réduite à une simple énumération de faits divers ou militaires. Les actes émanant des institutions Politiques ou ligueuses, d'Henri IV ou des alliés de la Ligue, ont une importance certaine pour une appréciation concrète des problématiques de l'époque, pour la compréhension des

52 idem, p. 29. Voir également M. Penzi, "L'histoire tragique et mémorable de Claude de Sainctes, évêque d'Evreux", dans Cahiers du CRH, n44, (2009), p. 9-26. 
partis, des idées politiques, des réelles questions qui vont bien au-delà de la simple succession du roi de Navarre au trône de France.

L'analyse des textes de l'été 1591 montre la portée des arrêts parlementaires et leur valeur politique et religieuse allant des dissidences schismatiques au consensus nécessaire à l'affirmation de la nouvelle monarchie. Les Ligueurs avaient compris cette portée. Et ils avaient répondu durement. Cette compréhension, qui semble claire aux yeux des contemporains, a été un peu sous-estimée par l'historiographie, ou encore tout simplement acceptée comme une forme de violence de la discussion politique de l'époque. Il est certain que les historiens ont mal estimé la portée réelle d'un acte, voire d'un ensemble d'actes, faits par les Parlements, qui avaient les soutiens des protestants du parti royal, et qui indiquaient clairement une future ligne de conduite politico-religieuse à la France gouvernée par Henri IV et ses Parlements.

Cette remarque est encore plus importante, car c'est à la lumière de cette piste de recherche, de cette analyse des écrits, des réponses et des invectives, qu'on comprend pleinement la signification des décisions prises par l'assemblée du clergé catholique royaliste, tenue sous la présidence du cardinal de Bourbon. En dépit de ses fautes formelles que Zampini montre du doigt, cette assemblée avait essayé de trouver une solution qu'on pourrait aisément appeler de compromis, vouée à trouver un dénouement avec Rome, aux problèmes encourus entre les catholiques ligueurs et leurs alliés d'un côté, et les catholiques royalistes de l'autre. L'assemblée avait donc préconisé l'envoi d'une députation à Rome, avec le but affiché d'informer le pape sur la situation française réelle. Il s'agissait, contrairement aux décisions du Parlement de Tours exposées plus haut, de ne pas couper définitivement les ponts avec Rome. En d'autres termes, le clergé gallican était conscient que le Parlement de Tours avait formellement déclaré un schisme avec Rome $^{53}$. Les prélats royalistes réunis en assemblée ont refusé de porter sur leurs épaules la faute d'une telle responsabilité. L'assemblée s’aperçoit qu'un éloignement de Rome et l'impossibilité de communiquer avec le pape aurait fait sombrer la France d'un point de vue religieux. Par ailleurs, cela présageait une sorte de schisme qui réduisait la probabilité d'une conversion d'Henri IV au catholicisme. La France séparée de Rome, la promesse de se convertir du souverain, encore affirmée à Mantes en juillet 1591, devenait lettre morte. Il ne pouvait y avoir de conversion au catholicisme si l'Église de référence se déclarait autocéphale. En outre, le texte ouvre la porte à la négociation, proposant de renseigner le pape qui connaît mal la situation.

53 Duplessis-Mornay, dans son discours envoyé à Henri IV, prononcé auparavant au Parlement de Tours, avait remarqué que cette députation à Rome était contraire aux arrêts parlementaires de l'été précédent. 
L'histoire demeura sans suite pour le cardinal de Bourbon et les évêques de l'assemblée de Chartres. En réalité, Grégoire XIV était mort au moment de l'entrevue. Son successeur ne resta en charge que deux mois, le nouveau pape Clément VIII n'ayant été élu que fin janvier 1592. Ce dernier adopta une politique différente de celle de son prédécesseur Grégoire XIV. Mais son soutien à la Ligue fut aussi fort que celui apporté les années précédentes. En décembre 1591, dans le conflit entre les Parlements et les prélats royalistes catholiques, les parlementaires avaient gagné la première manche, même si la dernière affirmation du cardinal de Bourbon laissait entrevoir une ouverture auprès du monarque, pour contraindre laïcs et juristes à s'occuper d'affaires qui ne concernaient pas le spirituel. Une deuxième manche fut jouée plus tard en 1592. Mais les temps avaient changé par rapport à l'été 1591 . Une nouvelle politique avait été mise en marche par Henri IV, et le cardinal de Gondi en était, avec son voyage à Rome, la première et peut être la plus éclatante manifestation aux yeux des catholiques tous partis confondus ${ }^{54}$.

54 R. de Maio, "Alessandro Franceschi e il Cardinale Pierre Gondi nella riconciliazione di Enrico IV”, dans Eugène Tisserant (éd.) : Mélanges. Città del Vaticano : Biblioteca Apostolica Vaticana, 1964, vol. IV, p. 313-356. 